\title{
El crecimiento de maestrías y doctorados según el Plan de Desarrollo Estratégico del Sistema de Educación Superior de Honduras 2014-2023
}

\section{The expected growth of graduate programs according to the Strategic Development Plan for Higher Education in Honduras 2014-2023}

Claudio Fernando Salgado Chavarría*

claudio.student@gmail.com

\section{Resumen}

El objetivo de este ensayo es indagar acerca del crecimiento de maestrías y doctorados que se estipula en el Plan de Desarrollo Estratégico del Sistema de Educación Superior de Honduras 2014-2023. Primero, hacemos una exposición sucinta del contenido y objetivos estratégicos del Plan. Seguidamente, describimos los objetivos y las acciones que versan sobre maestrías y doctorados. A continuación, examinamos los avances de dichos programas a 2016, tanto en cantidad como en normativas emitidas respecto a esos grados académicos. El ensayo finaliza con un planteamiento acerca de la importancia de la formación de docentes para el Plan de Desarrollo Estratégico y con sugerencias en ese sentido.

Palabras clave: desarrollo estratégico, educación superior, planificación, posgrados

\footnotetext{
* Docente de la Universidad Católica, Honduras.

Recibido 31 de agosto de 2018 / Aceptado 8 de noviembre de 2018 DOI: https://dx.doi.org/10.5377/paradigma.v25i40.7534

$<$ Paradigma> Revista de Investigación Educativa 40 Noviembre 2018/ ISSN: 1817-4221

Los articulos de la Revista Electrónica Paradigma del Instituto de Investigación y Evaluación Educativas y Sociales de la Universidad Pedagógica Nacional Francisco Morazán, de Honduras, se comparten bajo términos de la Licencia Creative Commons: Se permite que otros puedan descargar las obras y compartirlas con otras personas, siempre y cuando se 


\section{Abstract}

This essay aims to explore the expected growth of graduate programs according to the Strategic Development Plan for Higher Education in Honduras 2014-2023. First, I explain the topics and the strategic aims of the Plan. Then, I describe the objectives and actions that deal specifically with master and $\mathrm{PhD}$ programs. Next, I acknowledge that there has been progress of these programs to 2016, both in terms of quantity and regulations concerning those academic degrees. The essay ends with a statement on the importance of the training of professors to implement the Strategic Development Plan and with suggestions on this matter.

Keywords: graduate programs, higher education, planning, strategic development

\section{Ensayo}

\section{El plan de desarrollo estratégico: introducción y desafíos}

El Plan de Desarrollo Estratégico del Sistema de Educación Superior de Honduras 2014-2023 (Dirección de Educación Superior, 2014) es el documento que hoy en día marca la ruta para el conjunto de las universidades del país. Se aprobó en sesión del Consejo de Educación Superior el 21 de febrero de 2014. Su cumplimiento es obligatorio para todas las instituciones de educación superior (IES) nacionales y su vigencia finaliza en 2023.

Este Plan de Desarrollo del sector universitario se compone de cuatro capítulos: el Capítulo I ofrece un panorama mundial y nacional sobre las tendencias en 
educación superior; el Capítulo II se dedica a presentar el estado de la enseñanza universitaria en Honduras; el Capítulo III constituye propiamente el Plan de Desarrollo Estratégico, en el que se contienen los más importantes principios, objetivos y acciones estratégicas que regirán el conjunto del nivel universitario; el Capítulo IV representa una articulación entre estrategia y objetivos, puesto que detalla las maneras en que se adecuará dicho Plan y los modos con los que se pondrá en funcionamiento. Finalmente, en el documento se plasman los mecanismos de seguimiento y evaluación de la estrategia, los objetivos y los planes antes elaborados. El mismo Plan fija que cada seis meses se debe informar su avance a la Dirección de Educación Superior y a los demás órganos rectores del sistema (Dirección de Educación Superior, 2014).

Tras el estudio del entorno nacional, en el citado Capítulo III, se expresa que la «Estrategia Global del Sistema de Educación Superior de Honduras sea la siguiente: amplia diferenciación en calidad y acceso a la educación superior» (Dirección de Educación Superior, 2014, p. 44).

Para ejecutar esa estrategia, las IES han definido trece objetivos estratégicos, que se detallan en la siguiente tabla:

Los articulos de la Revista Electrónica Paradigma del Instituto de Investigación y Evaluación Educativas y Sociales de la Universidad Pedagógica Nacional Francisco Morazán, de Honduras, se comparten bajo términos de la Licencia Creative commons: Se permite que otros puedan descargar las obras y compartirlas con otras personas, siempre y cuando se 
Tabla 1

Estrategia global y objetivos estratégicos del Plan de Desarrollo Estratégico

Estrategia global: Amplia diferenciación en calidad y acceso

a la educación superior

№

Objetivo estratégico

1 Promover e impulsar el crecimiento armonioso e integral del Sistema

de Educación Superior, para fortalecer el desarrollo del país.

2 Aumentar la cobertura de educación superior al promedio latinoamericano, con equidad poblacional, regional, étnica y con atención a la población con necesidades especiales.

3 Ampliar y diversificar la oferta académica a nivel de grado y postgrado, en carreras orientadas fundamentalmente al desarrollo de la ciencia y la tecnología.

4 Elevar la oferta académica en el nivel de postgrados en sus diferentes estadios: especialidades, subespecialidades, maestrías y doctorados.

5 Promover la investigación y la vinculación de las instituciones de Educación Superior con la sociedad.

6 Promover el fortalecimiento, desarrollo y uso educativo de las Tecnologías de la Información y Comunicación en todos los ámbitos de la Educación Superior, para ampliar el acceso a oportunidades de aprendizaje, mejorar los logros de aprendizaje y calidad de la educación incorporando métodos avanzados de enseñanza-aprendizaje, así como reducir la brecha digital y favorecer la equidad en la educación superior. 
7 Implementar el Sistema de Evaluación, Acreditación y Certificación de la Calidad de la Educación Superior.

8 Fomentar el desarrollo de estructuras administrativas y de gestión académica curricular en los Centros de Educación Superior como parte de su planificación y gestión estratégica.

9 Promover en las instituciones de educación superior la elaboración de su Plan Estratégico y definición de su Modelo Educativo, basado en su filosofía, misión, visión, sus objetivos y su finalidad.

10 Promover el Programa de Formación Permanente, dirigido a personal directivo y docente de las instituciones de Educación Superior.

11 Revisar, desarrollar y aprobar nuevos estándares para apertura de centros de educación superior, sedes y carreras, acorde con las necesidades y a los criterios internacionales.

12 Actualizar las normas académicas de la educación superior de Honduras para armonizarlas con las tendencias de homologación/armonización de estándares, títulos, currículos, etc., en la región centroamericana.

13 Certificar la gestión y calidad del sistema de educación superior.

Nota. Fuente: Elaboración propia con base en Dirección de Educación Superior (2018)

En la práctica, la estrategia global constituye una apuesta por duplicar la matrícula del nivel terciario de la educación, del 15\% al 30\% de la población hondureña entre 18 y 24 años. En términos absolutos, este incremento significa que se espera que los centros de educación superior estén aptos para recibir en torno a 150 mil alumnos más que en 2012 (Dirección de Educación

Los articulos de la Revista Electrónica Paradigma del Instituto de Investigación y Evaluación Educativas y Sociales de Universidad Pedagógica Nacional Francisco Morazán, de Honduras, se comparten bajo términos de la Licencia Creative Commons: Se permite que otras puedan descargar las obras y compartirlas con otras personas, siempre y cuando se reconozca su autoria, pero na se pueden cambiar de ninguna manera ni se pueden utilizar comercialmente. 
Superior, 2014). Lo más interesante de dicha apuesta consiste en que la admisión a los centros universitarios deberá ser racionalizada, puesto que tendrá que priorizar a poblaciones tradicionalmente marginadas, como etnias o personas con diversidad funcional, y a regiones que -a 2012-no contaban con facilidades de este nivel educativo (Dirección de Educación Superior, 2014).

Con respecto a la diferenciación en calidad, las IES se han comprometido a que esta se certifique por parte de una firma especializada extranjera. La certificación del sistema irá acompañada de mejoras a lo interno de cada institución, con énfasis en la profesionalización de los cuerpos administrativos y de planificación y en la puesta en funcionamiento de herramientas que contribuyan a perfeccionar el manejo de cada universidad.

El compromiso adoptado por las IES significa un reto de grandes magnitudes, en tanto que se busca incrementar el tamaño del sector a la vez que deberán generarse las capacidades para acoger a tal población. Se trata de aprovechar el bono demográfico del que se disfruta en la actualidad, además de que es una formidable manera para contribuir con muchas familias, que todavía ven en la educación universitaria un medio para la movilidad social y la mejora en sus condiciones de vida.

También hay que señalar que este Plan de Desarrollo Estratégico abona al cumplimiento de la Ley de Educación Superior. Esta legislación de finales de los 80, manda en su art. 31 que «periódicamente deberá elaborarse un Plan Nacional de Desarrollo de Educación Superior» (Dirección de Educación Superior, 1994). La relevancia de este tipo de acciones concertadas ha sido explicada por voces autorizadas en el campo educativo. Para el caso, Salgado (2001) advertía que la falta de planificación era una debilidad del sistema universitario de principios del siglo XXI y Calderón (2011) consideraba que un 
documento macro serviría de guía del «crecimiento y desarrollo del Sistema con altos niveles de calidad y pertinencia», a inicios de esta década.

Junto a los méritos y bondades del Plan de Desarrollo, existen retos a los que hay que atender. Ningún plan está exento de observaciones ni es imposible ofrecer argumentos para su mejora. Entre esos retos que identificamos está la formulación de la estrategia global.

En cuanto a ese reto, la estrategia global refiere al desarrollo del sector universitario en términos de calidad y acceso. Estos dos componentes son, de acuerdo con un informe del BID, las dos políticas públicas que más se siguen en el ámbito educativo latinoamericano (Stein et al., 2006, p. 241). Si de por sí es difícil seguir una de ellas, ya sea por los fondos para aumentar las capacidades institucionales o por la cantidad de documentación sobre el desempeño de la calidad, nos parece una gran hazaña que ambas políticas educativas vayan de la mano.

A efectos ilustrativos, piénsese en que la Dirección de Educación Superior deberá de disponer de personal, procedimientos, tecnología, sistemas de acreditación y presupuesto para llevar a cabo una supervisión efectiva. Mientras ocurre esa reorganización, las universidades estarán fortaleciendo sus unidades internas, estructurando sus sistemas de gestión y, al mismo tiempo, diversificando la oferta educativa, construyendo nuevas sedes y viendo las maneras en que pueden aumentar la cobertura.

A esto habría que añadir que se espera que el crecimiento sea con equidad, alcanzando a poblaciones históricamente excluidas de la educación superior y a zonas del país que no han tenido establecimientos universitarios. En cuanto al primer aspecto, las universidades sí se han expandido a otras ciudades, lo cual es buena señal del cumplimiento del Plan de Desarrollo Estratégico

Los articulos de la Revista Electrónica Paradigma del Instituto de Investigación y Evaluación Educativas y Sociales de la Universidad Pedagógica Nacional Francisco Morazán, de Honduras, se comparten bajo térrminos de la Licencia Creative Commons: Se permite que otros puedan descargar las obras y compartirlas con otras personas, siempre y cuando se 
(Dirección de Educación Superior, 2018). En cuanto al acceso, la Dirección de Educación Superior (2018, p. 69) expresa que: «se continua [sic] teniendo una cobertura baja si se compara con el resto de países de América Latina y existe una gran cantidad de jóvenes graduados de educación media que no acceden a estudios en educación superior». Pero no se ha informado si esa expansión es congruente con la calidad que se espera del nivel universitario ni si se ha incentivado la matrícula de poblaciones tradicionalmente abandonadas, como indígenas, jóvenes de escasos recursos, habitantes de zonas rurales o personas con diversidad funcional, tal y como estipula el Plan de Desarrollo Estratégico.

\section{Las maestrías y doctorados a la luz del plan de desarrollo estratégico}

Entre los objetivos que se desprenden de la estrategia global, se hallan algunos que versan sobre los más altos grados académicos, como son los objetivos tercero y quinto, los cuales determinan las áreas del saber que tendrán preferencia en los próximos años.

Sin embargo, el más claro acerca de los posgrados es el objetivo estratégico cuarto, que expresa lo siguiente: «Elevar la oferta académica en el nivel de postgrados en sus diferentes estadios: especialidades, sub-especialidades, maestrías y doctorados» (Dirección de Educación Superior, 2014, p. 28).

Para la consecución de dicho objetivo, el Plan de Desarrollo define cuatro acciones, a saber (Dirección de Educación Superior, 2014):

a. Realizar un estudio de la actual oferta académica de postgrado en el nivel superior, con el propósito de identificar prioridades y necesidades de formación; b. Elaboración de un Plan de Desarrollo para ampliar la oferta y para asegurar la pertinencia y calidad de los 
mismos; c. Clasificar los programas de postgrados en profesionalizantes y de investigación, y promover la creación de postgrados de investigación; $\mathrm{d}$. Ampliar la oferta de maestrías de 23\% que se tiene en 2013 a un mínimo al [sic] 30\% en el 2022 y los doctorados de un $1.3 \%$ a un mínimo del 10\% del total de la oferta del Nivel Superior.

Esto quiere decir que los entes coordinadores del sector universitario aspiran a realizar un diagnóstico sobre las potencialidades del país y los saberes que las IES todavía no cubren. Con base en ese diagnóstico, se pretende elaborar un documento más técnico sobre los campos prioritarios a nivel nacional, que vendrán a satisfacer tanto las demandas de formación de profesionales en la administración pública, la empresa privada y la sociedad civil, como la preparación de los futuros docentes de centros privados y estatales. Dentro de las acciones arriba señaladas, conviene mencionar la propuesta de clasificar la oferta educativa en profesionalizante o de investigación, lo que a primera vista resolvería algunas dificultades que Medina (2015) ha señalado en esta revista.

Los Planes Operativos Anuales también hacen referencia a los posgrados, ya que en el segundo objetivo estratégico -»aumentar la cobertura de educación superior al promedio latinoamericano con equidad poblacional, regional, étnica y con atención a la población con necesidades especiales» (Dirección de Educación Superior, 2014) - se formulan las siguientes actividades:

-Ampliar la oferta académica de conformidad con las prioridades de desarrollo del país; -Ampliar la cobertura geográfica de educación superior; -Diversificar las modalidades de estudio; [...]-Ampliar la oferta académica de maestrías como mínimo al 30\% y los doctorados

Los articulos de la Revista Electrónica Paradigma del Instituto de Investigación y Evaluación Educativas y Sociales de la Universidad Pedagógica Nacional Francisco Morazán, de Honduras, se comparten bajo térrminos de la Licencia Creative Commons: Se permite que otras puedan descargar las obras y compartirlas con otras personas, siempre y
reconozca su autoria, pero na se pueden cambiar de ninguna manera ni se pueden utilizar comercialmente. 
al 10\% del total de la oferta del Nivel Superior. (Dirección de Educación Superior, 2014)

En resumen, el porcentaje esperado para maestrías y doctorados sería el siguiente:

Tabla 2

Crecimiento de maestrías y doctorados según el Plan de Desarrollo

Estratégico

\begin{tabular}{lll}
\hline Grado & $\begin{array}{l}\text { Antes del Plan } \\
\text { de Desarrollo (\%) }\end{array}$ & $\begin{array}{l}\text { Según el Plan de } \\
\text { Desarrollo (\%) }\end{array}$ \\
\hline Grado asociado/ & & n.d. \\
Técnico universitario & 15 & n.d. \\
Licenciatura & 54 & n.d. \\
Especialidad/ Sub-especialidad & 7 & 30 \\
Maestría & 23 & 10 \\
Doctorado & 1 & 10 \\
\hline
\end{tabular}

Nota. $n$. d. = No definido. Fuente: Elaboración propia con base en Dirección de

Educación Superior (2014)

Esto quiere decir que las IES han contraído la obligación de que el crecimiento de los estudios de maestría y doctorado sea de alrededor de 7 y 9 puntos porcentuales (p.p.), respectivamente, las cuales son cifras nada despreciables.

Téngase en cuenta que el Plan de Desarrollo Estratégico se elaboró con datos de 2013, cuando había 388 carreras aprobadas. De estas, 93 eran maestrías y 5 eran doctorados (Dirección de Educación Superior, 2014). Si se mantuviera ese mismo total, a inicios de la próxima década se tendría la siguiente oferta de posgrado: 
El crecimiento de maestrías y doctorados según el Plan de Desarrollo

Estratégico del Sistema de Educación Superior de Honduras 2014-2023

Tabla 3

Estimación del crecimiento de maestrías y doctorados

\begin{tabular}{|c|c|c|c|c|}
\hline \multirow[b]{2}{*}{ Grado } & \multicolumn{2}{|c|}{$\begin{array}{l}\text { Antes del Plan de } \\
\text { Desarrollo (2013) }\end{array}$} & \multicolumn{2}{|c|}{$\begin{array}{l}\text { Según el Plan de } \\
\text { Desarrollo }\end{array}$} \\
\hline & № & $\%$ & $\mathrm{~N}^{\mathrm{o}}$ & $\%$ \\
\hline \multicolumn{5}{|l|}{ Grado asociado } \\
\hline /Técnico universitario & 58 & 15 & n. d. & n.d. \\
\hline Licenciatura & 205 & 53 & n. d. & n. d. \\
\hline Especialidad/Sub-especialidad & 27 & 7 & n. d. & n. d. \\
\hline Maestría & 93 & 24 & $116^{\mathrm{a}}$ & 30 \\
\hline Doctorado & 5 & 1 & $39^{\mathrm{a}}$ & 10 \\
\hline Total & 388 & 100 & $388^{\underline{a}}$ & 100 \\
\hline
\end{tabular}

Nota. n. d. = No definido. Fuente: Elaboración propia con base en Dirección de

Educación Superior (2014)

a Suponiendo que el total de carreras aprobadas para el nivel de educación superior se mantiene en 388 .

Bajo el supuesto de 388 carreras aprobadas por el Consejo de Educación Superior, en términos absolutos habrá más de 110 maestrías y 30 doctorados funcionando en 2023. Este crecimiento abre oportunidades para que las personas se especialicen y para que las empresas y el gobierno fortalezcan sus cuadros. También ofrece nuevos caminos para adentrarse en la ciencia y el desarrollo del conocimiento, tanto aplicado como fundamental.

\section{Avances y retos de los programas de maestría y doctorado}

Durante el primer semestre de este año, la Dirección de Educación Superior (2018) publicó el informe La educación superior en Honduras y sus líneas 
estratégicas de desarrollo. En este se presentan datos actualizados sobre los aspectos más relevantes de la educación nacional. En cuanto al nivel terciario, podemos observar que en 2016 hubo un aumento de más de 60 programas de estudio, en comparación con 2013.

Tabla 4

Aumento de la oferta académica en el periodo 2013-2016

\begin{tabular}{lll}
\hline Grado & $\begin{array}{l}\text { Antes del Plan de } \\
\text { Desarrollo (2013) } \\
\text { № }\end{array}$ & $\begin{array}{l}2016 \\
\text { № }\end{array}$ \\
\hline Grado asociado & 58 & 63 \\
/Técnico universitario & & \\
Licenciatura & 205 & 232 \\
Especialidad/Subespecialidad & 27 & 29 \\
Maestría & 93 & 125 \\
Doctorado & 5 & 8 \\
\hline Total & 388 & 457 \\
\hline
\end{tabular}

Nota. Fuente: Elaboración propia con datos de Dirección de Educación Superior

En términos porcentuales, la siguiente tabla muestra que las maestrías se van acercando a los objetivos del Plan de Desarrollo Estratégico, pues en 2016 alcanzan el 27\% de la oferta total. Por su parte, los doctorados empiezan a crecer, aunque a ritmo lento, ya que pasan del 1\% al $2 \%$ de los programas aprobados. 
El crecimiento de maestrías y doctorados según el Plan de Desarrollo

Estratégico del Sistema de Educación Superior de Honduras 2014-2023

Tabla 5

Avance de los programas de maestría y doctorado a 2016

\begin{tabular}{llll}
\hline Grado & $\begin{array}{l}\text { Antes del Plan de } \\
\text { Desarrollo } \\
(2013)(\%)\end{array}$ & $2016(\%)$ & $\begin{array}{l}\text { Según el Plan de } \\
\text { Desarrollo (\%) }\end{array}$ \\
\hline $\begin{array}{l}\text { Grado asociado/ } \\
\text { Técnico universitario }\end{array}$ & 15 & 14 & n. d. \\
Licenciatura & 53 & 51 & n. d. \\
Especialidad/ & 7 & 6 & n. d. \\
Subespecialidad & 24 & 27 & 30 \\
Maestría & 1 & 2 & 10 \\
Doctorado & 100 & 100 & 100 \\
\hline Total & & &
\end{tabular}

Nota. n. d. = No definido. Fuente: Elaboración propia con datos de Dirección de

Educación Superior (2018)

En cuanto a regulaciones del nivel de posgrado, ese mismo informe dice lo siguiente (Dirección de Educación Superior, 2018):

El objetivo tres aborda la importancia de elevar la oferta académica en el nivel de postgrados, al igual que los avances anteriores, también se cuenta con un Reglamento para la regulación y funcionamiento del Sistema de Estudios de Posgrado del Nivel de Educación Superior de Honduras, acuerdo n⿳0 3210-299-2016, con ello se pretende elevar la calidad y pertinencia de los programas.

En efecto, en los últimos años se emitió el nuevo reglamento de posgrados, que introduce innovaciones en el sistema de educación superior. Por ejemplo,

Los articulos de la Revista Electrónica Paradigma del Instituto de Investigación y Evaluación Educativas y Sociales de Universidad Pedagógica Nacional Francisco Morazán, de Honduras, se comparten bajo términos de la Licencia Creative Commons: Se permite que otros puedan descargar las obras y compartirlas con otras personas, siempre y cuando se reconozca su autoría, pero no se pueden cambiar de ninguna manera ni se pueden utilizar comercialmente. 
dicho reglamento clasifica a las maestrías en profesionalizantes o académicas en función de la duración de los estudios y el tipo de trabajo final.

Según el art. 18, el objetivo de las maestrías profesionalizantes es: «desarrollar una formación teórico-práctica, con competencias profesionales y que incorpora elementos investigativos, concluyendo con un trabajo final de graduación» (Consejo de Educación Superior, 2016). El plan de estudios estará conformado por al menos un 25\% de investigación aplicada, entre 40 y 50 créditos y se desarrollará en un tiempo mínimo de un año y máximo de dos, dependiendo de si se dicta a tiempo completo o parcial.

Las maestrías académicas se describen en el art. 23 de ese reglamento como: «un proceso de formación cuya naturaleza es esencialmente de investigación. Concluye con una tesis como requisito de graduación, requiriendo ésta una perspectiva teórica conceptual y una propuesta metodológica» (Consejo de Educación Superior, 2016). Estas tendrán una carga de al menos un 45\% para investigación, entre 50 y 60 créditos y su programa de estudios se extenderá por dos años.

Ambas maestrías prepararán para la docencia universitaria y efectuarán algún tipo de investigación, aunque para las primeras su orientación será aplicada y de generación de competencias laborales, mientras que las segundas serán tesis en las que primará el conocimiento y discusión del fundamento de las ciencias.

Otra diferencia sustancial radica en que las maestrías académicas permiten el acceso a los estudios doctorales, mientras que los graduados de maestrías profesionalizantes deberán de realizar una pasantía de un mínimo de seis meses para dotarse de herramientas con las puedan elaborar trabajos 
científicos. Las pasantías tendrán lugar en unidades académicas de las distintas universidades (Consejo de Educación Superior, 2016).

Respecto de los doctorados, el art. 27 del nuevo reglamento los define como: «estudios del más alto nivel académico-profesional, fundamentado en la investigación y caracterizado por la realización de un trabajo original e individual publicable, bajo la estrecha supervisión de un equipo de expertos calificados que evalúa continuamente el desempeño del candidato» (Consejo de Educación Superior, 2016).

En este nivel formativo se abre la posibilidad de doctorados que no exijan una maestría, ya que el mismo art. 27 expresa que: «El doctorado sobre la licenciatura [...] requiere como mínimo de 120 créditos y 140 créditos como máximo, de 5400 a 6300 horas académicas, tendrá una duración de cinco a ocho años» (Consejo de Educación Superior, 2016).

La tercera innovación yace en que se regula la apertura de estudios posdoctorales en el país, conforme con lo establecido en el Capítulo VIII del nuevo reglamento de posgrados. El posdoctorado no constituye otro grado universitario, sino que tiende a «favorecer nuevos desarrollos académicos de los graduados de doctorado, creando un ámbito estimulante para la docencia, la investigación de alto nivel y el intercambio científico» (Consejo de Educación Superior, 2016). En ese entorno de aprendizaje, los doctores podrán perfeccionar sus habilidades metodológicas, actualizarse en su área del saber y tendrán la oportunidad de redactar textos académicos.

Estas tres innovaciones tardarán algunos años en difundirse en el sistema de educación superior, debido a que todavía no se han implantado programas doctorales en los que solo haya que presentar el título de licenciatura, ni se ha solicitado la creación de posdoctorados. Además hay que considerar que,

Los articulos de la Revista Electrónica Paradigma del Instituto de Investigación y Evaluación Educativas y Sociales de la Universidad Pedagógica Nacional Francisco Morazán, de Honduras, se comparten bajo térrminos de la Licencia Creative Commons: Se permite que otros puedan descargar las obras y compartirlas con otras personas, siempre y cuando se 
según disposiciones del nuevo reglamento del Consejo de Educación Superior (2016), primero tendrá que aprobarse la reforma a la «Guía para la Elaboración de Planes de Estudio de Posgrado» y luego habrá un plazo para que entre en vigor la clasificación de las maestrías en profesionalizantes o académicas.

Tras el repaso de los últimos años, conviene insistir en que sí ha habido crecimiento en el número de planes de estudio de maestría y doctorado. Las universidades estatales y privadas han trabajado para ampliar las oportunidades de estudio en el país. Pero desconocemos si ese aumento obedece a la intención de seguir el Plan de Desarrollo o si es solo un avance natural del sistema de educación superior.

Los datos de la Dirección de Educación Superior tampoco permiten conocer si se va cumpliendo el Plan en relación con el acceso equitativo, concretamente en posgrado. Es decir, no se detalla si las instituciones universitarias han realizado actividades de comunicación y mercadotecnia a fin de priorizar la matrícula de quienes no suelen integrar las cohortes de maestría y doctorado. Estas personas enriquecerían la experiencia dentro del aula, ya que aportan miradas novedosas y perspectivas que no suelen considerarse en el temario de las diversas asignaturas.

El informe de 2018 tampoco deja claro si ese crecimiento de posgrados tuvo lugar en nuevas regiones del país. De ser así, sería una gran ayuda para que los estudiantes no se desplacen a las grandes urbes hondureñas y no inviertan más fondos para su manutención. También podría ser señal de que la universidad toma la iniciativa de relacionarse con municipios y mancomunidades, en lugar de esperar a que sean contactadas por autoridades subnacionales. 


\section{Los docentes de posgrado y el plan de desarrollo estratégico}

De todo lo que acabamos de exponer, nos parece que trasluce un elemento clave, aunque escasamente abordado en el Plan de Desarrollo y en el informe de 2018 de la Dirección de Educación Superior. Nos referimos a los docentes que se requerirán para conseguir los objetivos propuestos, específicamente en el nivel de posgrado. Véase que ambos documentos no explican cómo ni de dónde se obtendrán los profesionales que ejercerán las funciones más inherentes a la universidad, como son la docencia, la investigación y la extensión.

En cuanto a cifras disponibles, según Duriez, López, \& Moncada (2016), en 2014 había 9325 puestos docentes en el sistema de educación superior, que incluía a 508 destinados a programas de maestría y doctorado.

Tras unos años de ejecución del Plan de Desarrollo, se ha visto que el número de puestos docentes ha aumentado y se ha acercado a los diez mil; aunque, en términos relativos, el porcentaje destinado a impartir asignaturas y dirigir investigaciones en los más altos grados académicos se ha mantenido en poco más de $5 \%$.

Tabla 6

Incremento de los puestos docentes en el periodo 2014-2015

\begin{tabular}{lllll}
\hline Grado que imparten & \multicolumn{2}{l}{ Puestos docentes (2014) } & \multicolumn{2}{c}{ Puestos docentes (2015) } \\
\cline { 2 - 5 } Go & No & $\%$ & № & $\%$ \\
\hline Técnico universitario & 100 & 1 & 132 & 1 \\
Licenciatura & 8558 & 92 & 9004 & 92 \\
Especialidad/ & & & & \\
Subespecialidad & 159 & 2 & 178 & 2 \\
Maestría & 502 & 5 & 470 & 5 \\
Doctorado & 6 & 0 & 4 & 0 \\
\hline Total & 9325 & 100 & 9788 & 100 \\
\hline
\end{tabular}

Nota. Fuente: Elaboración propia con datos de Duriez, López, \& Moncada (2016) y de Dirección de Educación Superior (2018)

Los articulos de la Revista Electrónica Paradigma del Instituto de Investigación y Evaluación Educativas y Sociales de la Universidad Pedagógica Nacional Francisco Morazán, de Honduras, se comparten bajo términos de la Licencia Creative Commons: Se permite que otros puedan descargar las obras y compartirlas con otras personas, siempre y cuando se 
En virtud de la relación contractual con las IES, los datos para el periodo de vigencia del Plan de Desarrollo muestran que el nivel terciario cuenta entre $41-49 \%$ de puestos docentes a jornada completa y entre $59-51 \%$ de puestos docentes a jornada parcial o por hora.

Tabla 7

Tipo de jornada de puestos docentes en el periodo 2014-2015

\begin{tabular}{llccc}
\cline { 2 - 4 } Jornada & № & $\%$ & № & $\%$ \\
\hline Completa & 3815 & 41 & 4815 & 49 \\
Parcial & 1652 & 18 & 1373 & 14 \\
Por hora & 3858 & 41 & 3600 & 37 \\
Total & 9325 & 100 & 9788 & 100
\end{tabular}

Nota. Fuente: Elaboración propia con datos de Duriez, López, \& Moncada (2016) y de

Dirección de Educación Superior (2018)

Para propósitos de este ensayo, sería interesante saber cuántos docentes en activo tienen los grados académicos necesarios para apuntalar el crecimiento de las maestrías y doctorados que menciona el Plan de Desarrollo. El informe de este año de la Dirección de Educación Superior (2018) no recoge ese dato, pero al menos existe una estimación hecha por Duriez, López, \& Moncada (2016). Estos autores conjeturan que el 5\% de todos los profesores universitarios con jornada completa posee al menos un posgrado, que en valores absolutos representa un total de 191 en 2013. (Duriez, López, \& Moncada, 2016). 
La pregunta que surge es si esos docentes serán suficientes para alcanzar el esperado incremento de maestrías y doctorados en 7 y 9 puntos porcentuales (p.p.), respectivamente.

Concretamente para másteres, se demandarán perfiles diferenciados según la clasificación del nuevo reglamento: los programas profesionalizantes solicitarán que los docentes cuenten con grados iguales o superiores al que imparten, además de experiencia laboral relevante que pueda transmitirse a los alumnos. Por su parte, los programas académicos exigirán profesores con los más altos grados universitarios, así como con conocimientos actualizados y con investigaciones publicadas.

A esto habría que añadir que tampoco se han determinado las fuentes de donde saldrán los recursos que necesitarán estos profesionales, como acervos bibliográficos, fondos para publicación de textos, laboratorios y reactivos, becas para actualizarse, cursos para hacer uso de la tecnología, etc.

La pregunta se vuelve aún más compleja cuando -en conformidad con el Plan de Desarrollo- tenemos en cuenta la promesa de extender los estudios a regiones que no han gozado de centros universitarios. Si en las grandes ciudades suele ser un problema cubrir vacantes para ciertas áreas del saber, ¿de dónde provendrán los docentes para las zonas rurales y más alejadas del país? En ese caso, las IES están obligadas a realizar una exhaustiva búsqueda de talento humano, aunque habrá riesgos que comprometan la calidad, debido a que es muy difícil que encuentren docentes capacitados en localidades donde no ha habido presencia universitaria.

Por la importancia que tienen los docentes para sacar adelante el Plan de Desarrollo Estratégico, por las limitadas estadísticas oficiales y porque creemos

Los articulos de la Revista Electrónica Paradigma del Instituto de Investigación y Evaluación Educativas y Sociales de la Universidad Pedagógica Nacional Francisco Morazán, de Honduras, se comparten bajo térrminos de la Licencia Creative Commons: Se permite que otras puedan descargar las obras y compartirlas con otras personas, siempre y
reconozca su autoria, pero na se pueden cambiar de ninguna manera ni se pueden utilizar comercialmente. 
que harán falta para educar a las nuevas generaciones, vemos necesario sugerir tres maneras por las cuales las IES pueden dotarse de estos profesionales. La primera sugerencia versa sobre el apoyo en el Reglamento para la aprobación $y$ desarrollo de planes de estudios de grado y posgrado en convenio con universidades extranjeras (Consejo de Educación Superior, 2015). Como su nombre lo indica, es un mecanismo del sistema universitario por el que se alienta a trabar relaciones con universidades foráneas, con el objeto de que estas dicten programas propios y de que se creen programas conjuntos en Honduras.

Para su realización, las IES hondureñas deben suscribir convenios con centros extranjeros y, una vez elaborado el plan de estudios, se debe de enviar a la Dirección de Educación Superior para su examen y, de resultar favorable, su aprobación.

Este reglamento no incluye secciones sobre maestrías y doctorados, aunque en su interior sí hay disposiciones que de una u otra forma les conciernen. Por ejemplo, el art. 11 manda que las IES hondureñas diseñen instrumentos que monitoreen el desarrollo de los estudios bajo convenio y que evalúen la calidad percibida por los alumnos. También se concede que las prácticas profesionales y los trabajos de graduación se hagan incluso fuera del territorio nacional, a la luz del art. 19. Por otro lado, en el art. 21 se abre la posibilidad de que las carreras entre universidades locales y extranjeras se dicten de manera presencial, a distancia o virtual.

El reglamento también ordena que el título sea extendido por el centro foráneo, cuando se trate de estudios que ya se ofrecen en su campus. De igual forma, se menciona que los estudiantes-que podrían ser maestrandos o doctorandosadquieren los mismos derechos y obligaciones de los alumnos de la institución 
foránea. El claustro puede estar compuesto parcial o totalmente por docentes extranjeros, a tenor de los Capítulos VI y VII.

Otros dos puntos importantes para maestrías y doctorados son, en primer lugar, que los programas deberán de cumplir con el marco jurídico y normativo hondureño en lo referente a duración, cantidad de créditos y pertinencia según el contexto del país. En segundo lugar, las universidades de Honduras han de tener presente que los documentos que suscriban tienen una caducidad programada, dado que el reglamento limita a tres las promociones que podrán realizarse por convenio (Consejo de Educación Superior, 2015).

La segunda sugerencia se centra en los docentes con los que ya cuenta el sistema de educación superior. Se trata de una idea planteada por Salomón (2016), consistente en el aprovechamiento de profesores en activo para impartir cursos y asesorar trabajos de posgrado. Según la autora, en las universidades hondureñas existen profesionales que, de derecho, no han obtenido los más altos grados académicos, aunque, de hecho, han mantenido una producción intelectual continua y se han dedicado a líneas de investigación por años.

La idea de tener en consideración las publicaciones y la experiencia académica -en lugar del título- no es del todo novedosa, ya que según la autora se ha puesto en práctica en la Universidad Nacional Autónoma de Honduras (UNAH), como explica a continuación (Salomón, 2016):

Este dominio [de la teoría y la metodología de un campo del saber] se puede adquirir en el aula universitaria, vía obtención de grados académicos, o vía experiencia profesional, enseñanza superior e investigación rigurosa de un tema específico, algo que se refleja muy

Los articulos de la Revista Electrónica Paradigma del Instituto de Investigación y Evaluación Educativas y Sociales de la Universidad Pedagógica Nacional Francisco Morazán, de Honduras, se comparten bajo términos de la Licencia Creative Commons: Se permite que otras puedan descargar las obras y compartirlas con otras personas, siempre y
reconozca su autoria, pero na se pueden cambiar de ninguna manera ni se pueden utilizar comercialmente. 
bien en el Estatuto del Docente Universitario, que permite la reclasificación de profesores por cualquiera de las dos vías.

Esa política laboral es muy importante porque está respaldada por la UNAH, que, como bien se sabe, es la institución educativa más grande a nivel nacional y la que tiene la potestad constitucional de organizar y dirigir la educación superior. Al ser decana de las universidades, acumula experiencias que resultan valiosas para el resto de centros educativos, como la que acabamos de describir. El apoyo en docentes que no cuenten con posgrados, aunque sí tengan una sólida carrera académica e investigadora, puede ser una medida que podría adoptar el sistema de educación superior mientras se resuelven los problemas de contratación en ciertas áreas del saber. Estos docentes pueden compartir su pericia en aspectos metodológicos y teórico-conceptuales, en los temas que llevan estudiando por años y en los que avistan que tendrán futuro a nivel nacional e internacional.

Con esta medida, también se atajaría otro de los problemas más comunes en las maestrías y doctorados, como es la falta de personal calificado para dirigir trabajos. Según ilustra la misma autora («Un análisis del nuevo Reglamento de Posgrado de Educación Superior», 2017, p. 6),

¿Cuál es el componente de investigación en una maestría? ¿Cómo está estructurado? Esto requiere revisar cómo está establecida la selección del tema de investigación [...], así como el hecho de que los estudiantes tienen que seleccionar de esas líneas de investigación la de sus tesis; e incluso que es el posgrado el obligado a encontrarle el asesor que sea de la competencia del tema seleccionado. [...] El problema de encontrar los asesores de tesis lo tenemos que asumir tanto las universidades públicas como las privadas. 
La tercera sugerencia gira en torno a las incorporaciones de títulos de maestría o doctorado. Estas validaciones de estudios realizados en el extranjero no se tienen en cuenta ni en el Plan de Desarrollo Estratégico ni en La educación superior en Honduras y sus líneas estratégicas de desarrollo, aunque debe prestárseles atención porque pueden ser una fuente de profesores para los diversos niveles universitarios.

Específicamente para maestrías y doctorados, hemos recogido datos para el periodo 2014-octubre de 2017. Es decir, para los tres primeros años de vigencia del Plan de Desarrollo Estratégico. Durante este periodo tuvieron lugar 306 incorporaciones de títulos de maestría y 30 incorporaciones de títulos de doctorado.

Respecto a las maestrías, en la tabla que se presenta a continuación puede verse que casi un tercio de las incorporaciones pertenecen al grupo de «Ciencias sociales, educación comercial y derecho», que engloba carreras como administración, mercadotecnia, psicología o derechos humanos. Tras este, se hallan los grupos de «Ingeniería, industria y construcción» y de «Salud y servicios sociales», en donde se reúnen títulos como ingeniería sanitaria, ingeniería eléctrica, procesamiento de alimentos, nutrición o disciplinas médicas.

Tabla 8

Incorporaciones de títulos de maestría (2014-octubre 2017)

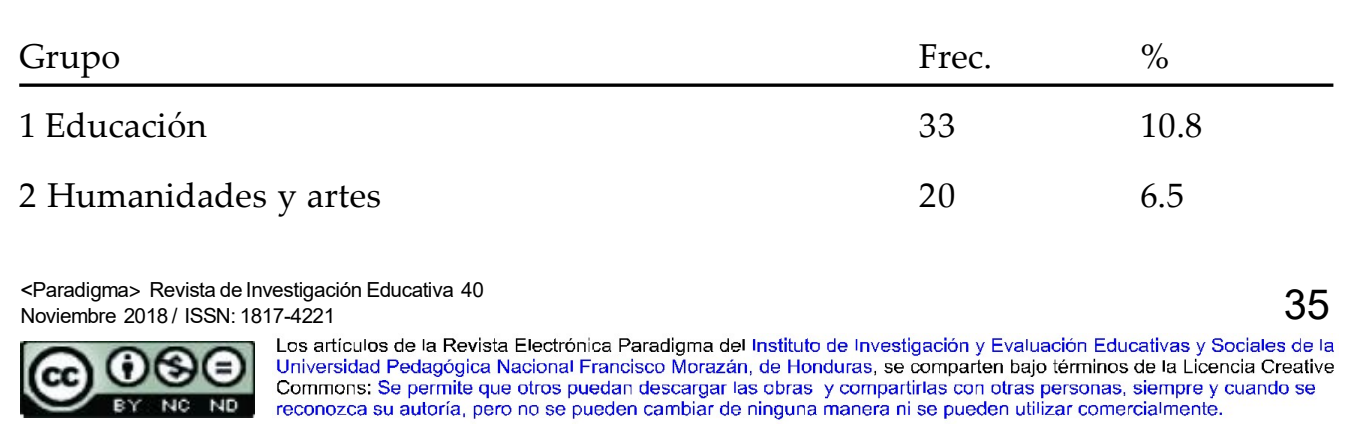




\begin{tabular}{lll}
3 Ciencias sociales, educación comercial y derecho & 97 & 31.7 \\
4 Ciencias & 37 & 12.1 \\
5 Ingeniería, industria y construcción & 41 & 13.4 \\
6 Agricultura & 30 & 9.8 \\
7 Salud y servicios sociales & 40 & 13.1 \\
8 Servicios & 8 & 2.6 \\
\hline Total & 306 & 100
\end{tabular}

Nota. Fuente: Dirección de Educación Superior y UNESCO (2013)

Por su parte, las incorporaciones de títulos de doctorado nos muestran que «Ciencias sociales, educación comercial y derecho» vuelve a situarse como el primer grupo. A este le siguen «Ciencias» $\mathrm{y}$ «Agricultura», en los que se encuentran titulaciones de doctor en matemáticas, química, producción agrícola o ganadera.

\section{Tabla 9}

Incorporaciones de títulos de doctorado (2014-octubre 2017)

\begin{tabular}{lll}
\hline Grupo & Frec. & $\%$ \\
\hline 1 Educación & 4 & 13.3 \\
2 Humanidades y artes & 3 & 10 \\
3 Ciencias sociales, educación comercial y derecho & 10 & 33.3 \\
4 Ciencias & 6 & 20 \\
5 Ingeniería, industria y construcción & 1 & 3.3 \\
6 Agricultura & 6 & 20 \\
7 Salud y servicios sociales & 0 & 0 \\
8 Servicios & 0 & 0 \\
\hline Total & 30 & 100 \\
\hline
\end{tabular}

Nota. Fuente: Dirección de Educación Superior y UNESCO (2013) 
Las cifras que acabamos de presentar para respaldar nuestra sugerencia han de examinarse con cautela, ya que apenas dejan entrever una parte de las personas que han obtenido posgrados en el extranjero y han vuelto al país. Téngase en cuenta que la incorporación no es un requisito para ejercer en empresas privadas, organismos internacionales ni en el sector público, salvo las universidades. Esta información proporcionada por la Dirección de Educación Superior tampoco permite saber si los que piden incorporar sus títulos de maestría o doctorado ya trabajan en instituciones de educación terciaria.

Sin embargo, nos parece que esas validaciones de estudio no deben de perderse de vista, puesto que alguna parte de esos profesionales no ha de laborar en el sistema de educación superior. Las IES podrían atraer a estos másteres y doctores mediante capacitaciones en teoría, metodología y, sobre todo, en didáctica. De todas maneras, los que se abocan a solicitar la incorporación representan una especie de talento potencial para el avance del sector educativo.

\section{Conclusiones}

En este ensayo hemos podido mostrar algunos resultados de la implementación del Plan de Desarrollo Estratégico del Sistema de Educación Superior. Específicamente en el nivel de posgrado, observamos incrementos en la oferta de maestrías y doctorados, de $24 \%$ a $27 \%$ y de $1 \%$ a $2 \%$, respectivamente, del total de los estudios universitarios en Honduras. Esto trae beneficios para el país, como la posibilidad de ahondar en el conocimiento de distintos campos del saber y como la mejora del personal de empresas, la administración pública y las oenegés.

Los articulos de la Revista Electrónica Paradigma del Instituto de Investigación y Evaluación Educativas y Sociales de la Universidad Pedagógica Nacional Francisco Morazán, de Honduras, se comparten bajo térrminos de la Licencia Creative Commons: Se permite que otros puedan descargar las obras y compartirlas con otras personas, siempre y
reconozca su autoria, pero na se pueden cambiar de ninguna manera ni se pueden utilizar comercialmente. 
Pero los resultados alcanzados no impiden que haya dificultades relacionadas con la ejecución del Plan de Desarrollo Estratégico. Entre estas subrayamos la complejidad del sistema, puesto que las IES deben realizar actividades de acreditación y expansión de la cobertura geográfica, a la vez que atienden tareas ordinarias de gestión académica. Especial atención merece la Dirección de Educación Superior, que tiene que aumentar sus capacidades institucionales y lidiar con los problemas que eso trae aparejado, mientras debe de dar curso a las labores de secretaría del nivel de educación superior, como son las crecientes solicitudes para aprobar planes de estudio, la validación de títulos del extranjero, la custodia de archivos y el mantenimiento de estadísticas.

Un segundo aspecto yace en que deben emitirse nuevas regulaciones sobre los posgrados. Hacen falta normativas acerca de las pasantías de los másteres profesionalizantes que quieran acceder al doctorado y los trámites y condiciones para abrir posdoctorados. También persisten algunas dudas sobre las tecnologías de información para los estudios universitarios. A modo de ejemplo, no queda claro si las diferentes universidades hondureñas pueden solicitar la apertura de doctorados cuya enseñanza sea totalmente virtual.

De igual forma, hay que mejorar los esquemas de seguimiento al actual Plan de Desarrollo Estratégico. Como pudimos ver, existen elementos críticos sobre los que no se pueden emitir valoraciones inequívocas, puesto que no se dispone de datos. No hay indicadores oficiales acerca del número de docentes universitarios que poseen maestrías o doctorados, ni medidas acerca de colectivos históricamente excluidos. El monitoreo y la evaluación es fundamental para el ciclo de proyectos, además de que tiene un propósito formativo, pues sirve de marco para redactar informes en los que se consignan lecciones a los futuros encargados de planificación. 
En ese sentido, nos parece crucial que se involucre a los docentes en cada una de las etapas de próximos planes de desarrollo: durante su elaboración, en las revisiones intermedias, en la evaluación final y la publicidad de los logros. Al tenerse la educación superior como un sistema, debe de evitarse la tentación de que se deshumanice a los actores del proceso educativo, entre los que se hallan el personal administrativo, los alumnos y, por supuesto, los docentes. La búsqueda de la efectividad de los planes de desarrollo no debe de ser obstáculo para su participación en los asuntos que más les conciernen.

Para finalizar, consideramos que debe explorarse la matrícula de otras poblaciones que suelen dejarse de lado en las IES. De momento se nos ocurre la inclusión de personas fuera del rango de 18 a 24 años -en el que se enfoca el actual Plan-, como pueden ser naturalmente los mayores de esa edad, los profesionales que necesitan actualizaciones en habilidades para el trabajo, los que demandan capacitaciones cortas para reciclarse, eincluso para cambiar de sector económico, y los que necesitan un empuje extra para salir del desempleo. En resumen, todavía hay muchas oportunidades para que el sistema de educación superior crezca ampliamente y que lo haga bajo criterios de calidad y equidad, como aporte al desarrollo de la sociedad hondureña.

\section{Referencias}

Calderón, R. (2011). El crecimiento y desarrollo de la educación superior en Honduras, una perspectiva desde la UNAH. Innovación Educativa, 11 (57), 81-89.

Consejo de Educación Superior. (2015). Reglamento para la aprobación y desarrollo de planes de estudios de grado y posgrado en convenio con

Los articulos de la Revista Electrónica Paradigma del Instituto de Investigación y Evaluación Educativas y Sociales de la Universidad Pedagógica Nacional Francisco Morazán, de Honduras, se comparten bajo térrminos de la Licencia Creative Commons: Se permite que otros puedan descargar las obras y compartirlas con otras personas, siempre y
reconozca su autoría, pero no se pueden cambiar de ninguna manera ni se pueden utilizar comercialmente. 
universidades extranjeras. Recuperado de https://docplayer.es/ 57225009-Reglamento-para-la-aprobacion-y-desarrollo-de-planes-deestudios-de-grado-y-posgrado-en-convenio-con-universidadesextranjeras.html»

Consejo de Educación Superior. (2016). Reglamento para la regulación y funcionamiento del sistema de estudios de posgrado del nivel de educación superior en Honduras. Recuperado de https://des.unah.edu.hn/ dmsdocument/2610-reglamento-de-educacion-tecnica-y-de-postgrado

Dirección de Educación Superior. (1994). Ley de educación superior, Reglamento general de la ley, Normas académicas del nivel de educación superior. Recuperado de https://des.unah.edu.hn/ dmsdocument/577

Dirección de Educación Superior. (2014). Plan de Desarrollo Estratégico del Sistema de Educación Superior de Honduras. Recuperado de https:// des.unah.edu.hn/repositorio/plan-estrategico-del-ses-2014-2023/

Dirección de Educación Superior. (2018). Laeducación superior en Honduras y sus líneas estratégicas de desarrollo. Recuperado de http:/ /joom.ag/8ydY

Duriez, M., López, V., \& Moncada, G. (2016). Educación Superior en Iberoamérica: Informe 2016. Informe nacional: Honduras. Recuperado dehttp://www.cinda.cl/wp-content/uploads/2016/11/HONDURASInforme-Final.pdf

Medina, E. (2015). La investigación en los posgrados académicos y profesionalizantes en Honduras. Paradigma: Revista de Investigación Educativa, 21 (35), 33-52. 
Salgado, R. (2001). La Educación Superior en Honduras. En M. Solís, M. Chávez, R. Hernández, G. Moncada, D. Orellana (comps.). (2004). Investigación educativa en la UPNFM: 1990-2000, (1 ed., pp. 264-275). Tegucigalpa: Dirección de Investigación/Dirección de Posgrado.

Salomón, L. (29 de junio de 2016). Posgrados, experiencia e investigación científica. Presencia Universitaria. Recuperado de https:// presencia.unah.edu.hn/noticias/posgrados-experiencia-einvestigación-científica

Stein, E., Tommasi, M., Echebarría, K., Payne, M., Lora, E., \& Morrison, N. (coords.). (2006). La política de las políticas públicas: Progreso económico y social en América Latina. Washington: Banco Interamericano de Desarrollo.

Un análisis del nuevo Reglamento de Posgrado en Educación Superior. (Abril-mayo de 2017). Investigación y Ciencia, pp. 6-7.

UNESCO. (2013). Clasificación Internacional Normalizada de la Educación CINE 2011. Recuperado de http://unesdoc.unesco.org/images/0022/ 002207/220782s.pdf

Los articulos de la Revista Electrónica Paradigma del Instituto de Investigación y Evaluación Educativas y Sociales de Universidad Pedagógica Nacional Francisco Morazán, de Honduras, se comparten bajo términos de la Licencia Creative Commons: Se permite que otros puedan descargar las obras y compartirlas con otras personas, siempre y cuando se 
(cc) $\odot \bigoplus \begin{aligned} & \text { Los articulos de la Revista Electrónica Paradigma del Instituto de Investigación y Evaluación Educativas y Sociales de la } \\ & \text { Universidad Pedagógica Nacional Francisco Morazán, de Honduras, se comparten bajo términos de la Licencia Creative }\end{aligned}$

BY NC ND Commons: Se permite que otros puedan descargar las obras y compartirlas con otras personas, siempre y cuando se
reconozca su autoria, pero no se pueden cambiar de ninguna manera ni se pueden utilizar comercialmente. 